\title{
CRNDE silencing promotes apoptosis and enhances cisplatin sensitivity of colorectal carcinoma cells by inhibiting the Akt/mTORC1-mediated Warburg effect
}

\author{
WENYU YANG, YANCHUN WANG, CHUNHUI TAO, YUNHAI LI, SHAN CAO and XIQIAN YANG
}

Clinical College of Chinese Medicine, Hubei University of Chinese Medicine, Wuhan, Hubei 430065, P.R. China

Received October 26, 2021; Accepted November 19, 2021

DOI: $10.3892 / \mathrm{ol} .2022 .13190$

\begin{abstract}
Colorectal cancer (CRC) is one of the most prevalent gastrointestinal tumors worldwide, with a high mortality rate. The IncRNA colorectal neoplasia differentially expressed (CRNDE) is upregulated in CRC and is involved in regulating the apoptosis, proliferation, and drug sensitivity of CRC cells. However, the specific underlying mechanisms remain to be elucidated. The aim of the present study was to investigate the effects of CRNDE on the Warburg effect in CRC cells, as well as the associated mechanisms. The expression of CRNDE in HCT-116 cells was overexpressed or silenced by transfection. Apoptosis, cisplatin sensitivity, the Warburg effect, and Akt/mTOR activation were evaluated. The results demonstrated that CRNDE inhibition decreased the proliferation and increased the apoptosis and cisplatin sensitivity of HCT-116 cells. In addition, CRNDE inhibition attenuated the Warburg effect in HCT-116 cells, as verified by a decrease in ATP production, lactic acid levels, glucose uptake, and the expression of Warburg effect-related enzymes (GLUT1, LDHA, HK2, and PKM2). CRNDE inhibition also suppressed the activity of the Akt/mTORC1 pathway, as demonstrated by the decreased phosphorylation of Akt, S6K, S6, and mTOR and the increased phosphorylation of 4EBP-1 and EIF-4E. The CRNDE overexpression-induced increase in ATP and lactic acid levels and glucose uptake in HCT-116 cells was reversed by Akt and mTOR inhibitors. These findings indicate that CRNDE silencing promotes apoptosis and enhances cisplatin sensitivity in colorectal carcinoma cells, which may be mediated by the regulation of the Warburg effect via the Akt/mTORC1 pathway. The present study thus provides a potential strategy for the treatment of CRC.
\end{abstract}

Correspondence to: Dr Xiqian Yang, Clinical College of Chinese Medicine, Hubei University of Chinese Medicine, 16 Huangjiahu West Road, Hongshan, Wuhan, Hubei 430065, P.R. China E-mail: 3251@hbtcm.edu.cn

Key words: colorectal cancer, Warburg effect, Akt/mTORC1 pathway, drug sensitivity

\section{Introduction}

Colorectal cancer (CRC) is one of the most prevalent types of gastrointestinal tumors worldwide, ranking third in cancer incidence and second in mortality rate. CRC led to 191,000 deaths in 2015 in China (1), and in 2018, it was estimated that there were more than 180,0000 new cases of CRC and 881,000 cancer-related deaths (2). The main treatment methods for CRC include surgical resection, chemoradiotherapy, and chemotherapy, but the 5-year survival of patients remains low (3-5). Moreover, with the repeated use of chemotherapeutic drugs, CRC tumors are prone to develop drug resistance, ultimately resulting in failed chemotherapeutic regimens $(6,7)$. Thus, it is crucial to understand the mechanism of CRC to develop effective therapeutic strategies.

Long non-coding RNAs (IncRNAs) are a type of RNA that is abnormally expressed in various tumors, including hepatocellular carcinoma (8), pancreatic cancer (9), bladder cancer (10), and CRC (11). They are associated with many biological processes, including cell proliferation, apoptosis, metabolism, and drug resistance (12-14). The lncRNA colorectal neoplasia differentially expressed (CRNDE), which was initially found to be upregulated in colorectal adenomas and carcinomas, is transcribed from human chromosome 16 (15). Previous findings showed that CRNDE is involved in the occurrence and development of numerous cancers, such as CRC (16), hepatocellular carcinoma (17), and non-small cell lung cancer (18), and can act as a biomarker for specific malignant tumors $(19,20)$. CRNDE promotes the proliferation and inhibits the apoptosis of cervical cells by regulating the PI3K/Akt pathway, resulting in poor prognosis (21), and CRNDE inhibition increases the chemosensitivity of medulloblastoma cells (22). Accumulating evidence has shown that CRNDE is upregulated in CRC and is associated with the regulation of CRC cell apoptosis, proliferation, and drug sensitivity (23-25). However, the specific underlying mechanisms of CRNDE-mediated cancer processes need to be elucidated.

The Warburg effect is one of the main metabolic alterations in cancer that enhances tumor growth $(26,27)$. Previous findings have indicated that Akt/mTOR activation promotes the Warburg effect and tumorigenesis in non-small cell lung cancer (28). In addition, Yang and Chen found that CRNDE enhances the progression of tongue squamous cell carcinoma by regulating the PI3K/Akt/mTOR signaling pathway (29). 
However, whether CRNDE affects the Warburg effect in CRC cells by regulating Akt/mTOR signaling, thereby influencing associated biological processes of tumorigenesis, needs to be clarified. To address this, CRNDE was overexpressed or downregulated in the human colorectal carcinoma cell line HCT-116, via transfection to evaluate its effect on cell apoptosis, cisplatin sensitivity, the Warburg effect, and Akt/mTOR activity. The aim of the present study was to clarify the mechanism of CRNDE, provide new targets for CRC treatment, reduce the occurrence of tumor cell drug resistance in CRC treatment, improve the effectiveness rate of $\mathrm{CRC}$ treatment and patient quality of life.

\section{Materials and methods}

Cell culture. Human colorectal carcinoma cell lines, HCT-116 and LOVO, and the human normal colonic epithelial cell line NCM460 were purchased from the Shanghai Institutes for Biological Sciences, Chinese Academy of Science. HCT-116 and NCM460 cells were cultured in McCoy's 5A medium (Gibco, Thermo Fisher Scientific, Inc.) supplemented with $10 \%$ fetal bovine serum (FBS; Gibco, Thermo Fisher Scientific, Inc.). LOVO cells were cultured in F12K medium supplemented with $10 \%$ FBS. All cells were incubated at $37^{\circ} \mathrm{C}$ with $5 \% \mathrm{CO}_{2}$. After confluence reached $90 \%$, the cells were harvested and reverse transcription-quantitative polymerase chain reaction (RT-qPCR) was performed to detect the expression of CRNDE and experiments were performed in triplicate.

Cell transfection and treatment. The CRNDE overexpression vector pCDH-CMV-MCS-EF1-CopGFP-T2A-Puro (CRNDE sequence: forward, 5'-GCTCTAGATGTTGGCTGAAAT TCAT-3', reverse, 5'-CGGGATCCTTATAGTCTATAAACA GG-3') and interference vector pSICOR (short hairpin RNA sequence: 5'-GGTGTTAAGTGTGATGCTTCC-3') were supplied by Addgene (Cambridge). HCT-116 cells were seeded into 24 -well plates at a density of $5 \times 10^{5}$ cells/well. When the confluence reached $70-80 \%$, the cells were transfected with pCDH-CRNDE (CRNDE overexpression, CRNDE-OE), empty pCDH-CMV-MCS-EF1-CopGFP-T2A-Puro vectors (CRNDE-OE negative control, CRNDE-NC), pSICOR-shCRNDE (CRNDE interference, sh-CRNDE), or empty pSICOR vectors (sh-CRNDE negative control, sh-NC) using Lipofectamine ${ }^{\mathrm{TM}} 2000$ (Invitrogen, Thermo Fisher Scientific, Inc.) according to the manufacturer's instructions. Non-transfected HCT-116 cells served as the control. After $24 \mathrm{~h}$ of transfection, the transfection rate and effect of CRNDE on HCT-116 cell proliferation, apoptosis, the Warburg effect, and Akt/mTOR complex 1 (mTORC1) pathway activity were evaluated.

After $24 \mathrm{~h}$ of transfection, HCT-116 cells were treated with cisplatin (Sigma-Aldrich) at 5 or $10 \mu \mathrm{M}$ for 12,24 , 48, 72 and $144 \mathrm{~h}$. Cell counting kit-8 (CCK-8) assays were performed to evaluate the cisplatin sensitivity of cells. Activity of the Akt/mTORC1 pathway in HCT-116 cells was evaluated after $24 \mathrm{~h}$ of treatment with $10 \mu \mathrm{M}$ cisplatin. To verify the influence of the Akt/mTORC1 pathway on the regulatory effect of CRNDE on the Warburg effect, HCT-116 cells were treated with the Akt inhibitor AZD5363 (500 nM; MedChemExpress) and the mTOR inhibitor AZD2014 (5 mM; MedChemExpress) for $6 \mathrm{~h}$. Subsequent to 24-h transfection, ATP production, glucose uptake, and lactic acid levels were also measured.

$R T-q P C R$. Total RNA was extracted from HCT-116, LOVO, NCM460, and HCT-116 cells after $24 \mathrm{~h}$ of transfection using TRIzol (Ambion; Thermo Fisher Scientific, Inc.) and reverse-transcribed into cDNA. The collected cDNA was amplified by PCR using the following primer sequences: Forward, 5'-ATTCATCCCAAGGCTG-3', reverse 5'-CAA AGACCAACGGCTG-3'; GAPDH forward, 5'-CCACTCCTC CACCTTTG-3', reverse 5'-CACCACCCTGTTGCTGT-3'. $G A P D H$ was used as an internal control. Data were analyzed using the $2^{-\Delta \Delta \mathrm{Cq}}$ method (30).

CCK-8 assay. The effect of CRNDE on the proliferation and cisplatin sensitivity of HCT-116 cells was investigated using the CCK-8 assay. Harvested HCT-116 cells were seeded into 96-well plates at a density of $5 \times 10^{3}$ cells/well and cultured overnight at $37^{\circ} \mathrm{C}$ with $5 \% \mathrm{CO}_{2}$. After treatment, the cells were incubated with $10 \mathrm{ml}$ of CCK-8 solution (Bioswamp; Wuhan Bienle Biotechnology Co., Ltd.) for $4 \mathrm{~h}$ at $37^{\circ} \mathrm{C}$ with $5 \% \mathrm{CO}_{2}$. The optical density of wells was measured at $450 \mathrm{~nm}$ using a Multiskan FC apparatus (Thermo Fisher Scientific, Inc.).

Flow cytometry. Flow cytometry was performed to evaluate cell apoptosis using an Annexin V-fluorescein isothiocyanate (FITC)/propidium iodide (PI) apoptosis detection kit (BD Biosciences). Harvested HCT-116 cells $\left(5 \times 10^{5}\right)$ were centrifuged at $1,000 \mathrm{x} \mathrm{g}$ at $4^{\circ} \mathrm{C}$ for $5 \mathrm{~min}$ and resuspended in $1 \mathrm{ml}$ of phosphate-buffered saline (PBS; Bioswamp), followed by centrifugation at $1,000 \mathrm{x}$ a at $4^{\circ} \mathrm{C}$ for $5 \mathrm{~min}$. The cells were then resuspended in $200 \mathrm{ml}$ of binding buffer (Bioswamp) and stained with $10 \mu \mathrm{l}$ of Annexin V-FITC and $10 \mu \mathrm{l}$ of PI in the dark at $4^{\circ} \mathrm{C}$ for $30 \mathrm{~min}$. After adding $300 \mu \mathrm{l}$ of binding buffer, the cells were subjected to flow cytometry (Beckman Coulter, Brea, CA, USA) and analyzed using CytExpert software (Beckman Coulter, Inc.; version 2.0).

Hoechst-33258 staining. Hoechst 33258 staining was performed to evaluate apoptosis. After treatment, HCT-116 cells were fixed in $4 \%$ paraformaldehyde at $4^{\circ} \mathrm{C}$ for $10 \mathrm{~min}$. After two washes with PBS for $3 \mathrm{~min}$, the cells were stained with $2 \mathrm{ml}$ of Hoechst 33258 staining solution $(10 \mu \mathrm{g} / \mathrm{ml})$ at room temperature for $5 \mathrm{~min}$. Finally, the cells were photographed using an inverted fluorescence microscope (Leica).

Western blot analysis. Total proteins were extracted from HCT-116 cells using radioimmunoprecipitation assay lysis buffer (Bioswamp) and quantified using a bicinchoninic acid assay kit (Bioswamp). SDS-PAGE (12\%) was performed and the proteins $(20 \mu \mathrm{g})$ were separated and transferred onto polyvinylidene fluoride membranes (MilliporeSigma). After blocking with $5 \%$ skimmed milk powder at $37^{\circ} \mathrm{C}$ for $1 \mathrm{~h}$, the membranes were incubated for $1 \mathrm{~h}$ at room temperature with primary antibodies against B-cell lymphoma-2 (Bcl-2, 1:1,000, PAB30041), glucose transporter type 1 (GLUT1, 1:1,000, PAB30526), hexokinase 2 (HK2, 1:1,000, PAB30052), phosphorylated (p)-Akt (1:1,000, PAB30040), Akt (1:10,000, PAB30042), p-mTOR (1:1,000, PAB43425-P), mTOR (1:1,000, PAB46102), and GAPDH (1:1,000, PAB36269) purchased 
from Bioswamp, as well as those targeting cytochrome-C (cyt-c, 1:500, ab216971), cleaved-caspase 3 (1:500, ab32042), lactate dehydrogenase A (LDHA, 1:5,000, ab101562), pyruvate kinase M2 (PKM2, 1:1,000, ab137852), p-eukaryotic translation initiation factor 4E-binding protein 1 (p-4EBP-1, 1:2,000, ab60529), 4EBP-1 (1:5,000, ab2606), eukaryotic translation initiation factor 4E (EIF-4E, 1:5,000, ab32024), p-S6K (1:1,000, ab60948), S6K (1:5,000, ab32529), p-S6 (1:10,000, ab184551), and S6 (1:1,000, ab32132), purchased from Abcam (Cambridge, UK), followed by incubation with goat anti-rabbit IgG secondary antibody (1:20,000, Bioswamp, PAB160011) for $1 \mathrm{~h}$ at $4 \mathrm{C}$. An enhanced chemiluminescence kit (Pierce; Thermo Fisher Scientific, Inc.) was used to detect the specific bands and analyzed using Tanon GIS software (version 4.2; Tanon Science and Technology Co., Ltd.) using GAPDH as a control.

Biochemical assay. Biochemical assays were performed to evaluate ATP production (A095, Nanjing Jiancheng Bioengineering Institute), glucose uptake (KA4086, Abnova), and lactic acid level (A019-2, Nanjing Jiancheng Bioengineering Institute) in HCT-116 cells using corresponding commercial kits according to the manufacturer's instructions.

Statistical analysis. Data are presented as means \pm standard deviation. Differences in data were analyzed using one-way analysis of variance followed by Tukey's post-hoc test. Data were analyzed using SPSS software (v19.0; IBM Corp.). $\mathrm{P}<0.05$ was considered to indicate a statistically significant difference.

\section{Results}

CRNDE silencing inhibits proliferation and promotes apoptosis and cisplatin sensitivity of HCT-116 cells. As shown in Fig. 1A, the expression of CRNDE in HCT-116 and LOVO cells was higher than that in NCM460 cells. As HCT-116 cells exhibited the highest expression of CRNDE, they were selected for subsequent experiments.

To investigate the effect of CRNDE on CRC cells, CRNDE was overexpressed or inhibited in HCT-116 cells via transfection (Fig. 1B). The CCK-8 assay demonstrated that CRNDE inhibition suppressed the viability of HCT-116 cells, as indicated by the decrease in optical density (Fig. 2A). In addition, CRNDE inhibition (sh-CRNDE) promoted the apoptosis of HCT-116 cells, as verified by the increase in the apoptosis rate, detected by flow cytometry (Fig. 2B), and the bright blue cells (white arrows), detected by Hoechst-33258 staining (Fig. 2C). The expression of apoptosis-related proteins was detected using western blotting. CRNDE overexpression upregulated the anti-apoptotic protein $\mathrm{Bcl}-2$ while inhibiting the expression of the pro-apoptotic proteins Cyt-c and Cleaved-caspase 3, whereas CRNDE inhibition had the opposite effect (Fig. 2D). The results of western blotting were consistent with those of flow cytometry and Hoechst-33258 staining. After transfection, HCT-116 cells were treated with cisplatin at 5 or $10 \mu \mathrm{M}$. Compared to that with control cells, cisplatin decreased the optical density of HCT-116 cells (Fig. 2E). Compared to that of cells treated with cisplatin, the optical density of cells treated with cisplatin combined with CRNDE overexpression was increased, whereas that of cells treated with cisplatin combined
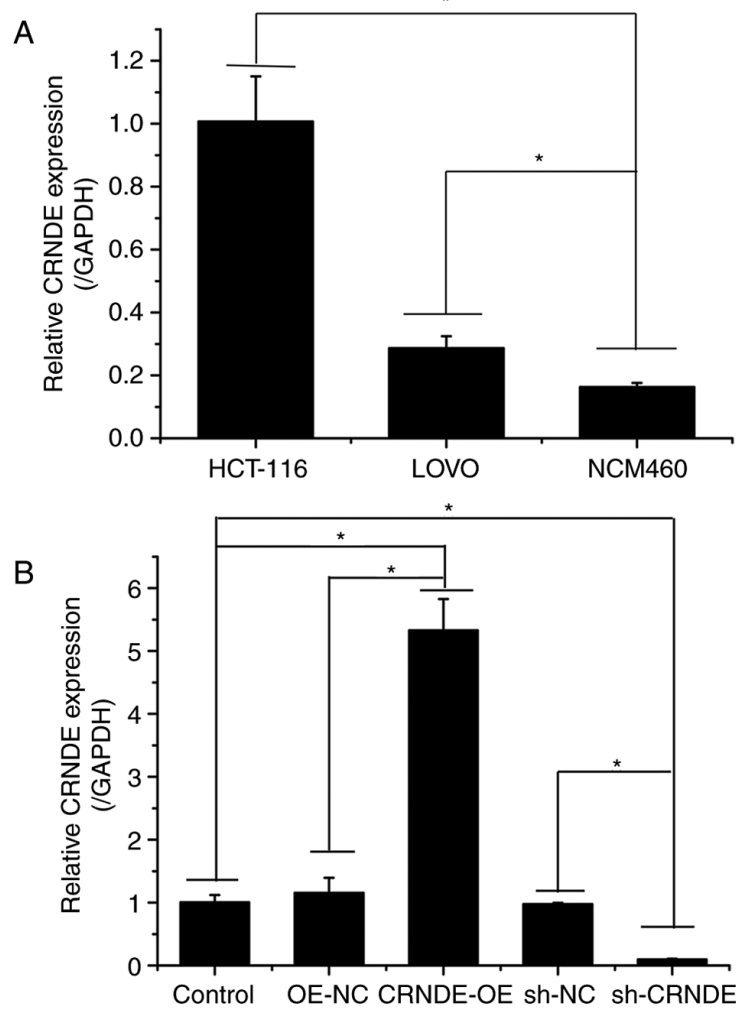

Figure 1. CRNDE is upregulated in HCT-116 cells. (A) The expression of CRNDE in HCT-116, LOVO, and NCM460 cells was detected by RT-qPCR. (B) The expression of CRNDE in transfected HCT-116 cells was detected by RT-qPCR. Data are presented as the mean $\pm \mathrm{SD}, \mathrm{n}=3,{ }^{*} \mathrm{P}<0.05$.

with CRNDE inhibition was decreased (Fig. 2E), indicating that CRNDE silencing decreased HCT-116 cell viability, and increased HCT-116 cell cisplatin sensitivity.

CRNDE silencing inhibits the Warburg effect in HCT-116 cells. Biochemical testing showed that compared to those in the control group, ATP and lactic acid levels and glucose uptake were increased in the CRNDE-OE group but decreased in the sh-CRNDE group (Fig. 3A). The expression of GLUT1, LDHA, HK2, and PKM2 was also detected by western blotting. CRNDE overexpression (CRNDE-OE) promoted the expression of GLUT1, LDHA, HK2, and PKM2, which was inhibited by CRNDE interference (sh-CRNDE) in HCT-116 cells (Fig. 3B). These results indicated that CRNDE silencing inhibited the Warburg effect in HCT-116 cells.

CRNDE silencing inhibits activation of the Akt/mTORC1 pathway in HCT-116 cells. Western blotting demonstrated that compared to that in the control cells, the protein expression of p-Akt, p-S6K, p-S6, and p-mTOR was increased in the CRNDE-OE group, whereas that of p-4EBP-1 and EIF-4E was decreased (Fig. 4). Conversely, the protein expression of p-Akt, p-S6K, p-S6, and p-mTOR was decreased in the sh-CRNDE group, whereas that of p-4EBP-1 and EIF-4E was increased (Fig. 4). These results indicate that CRNDE silencing inhibited the activation of the Akt/mTORC1 pathway in HCT-116 cells.

CRNDE silencing suppresses the Warburg effect in HCT-116 cells by inhibiting Akt/mTOR activation. To verify the 


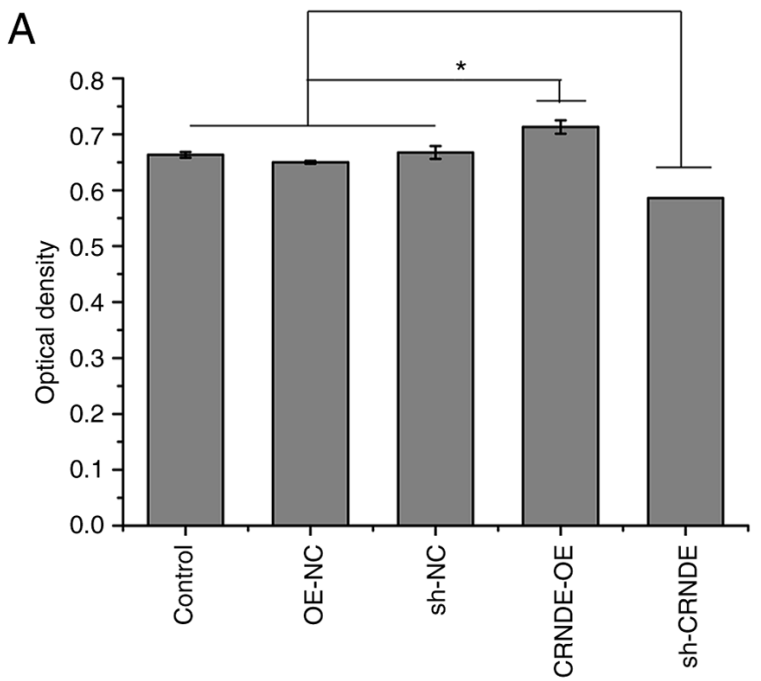

B
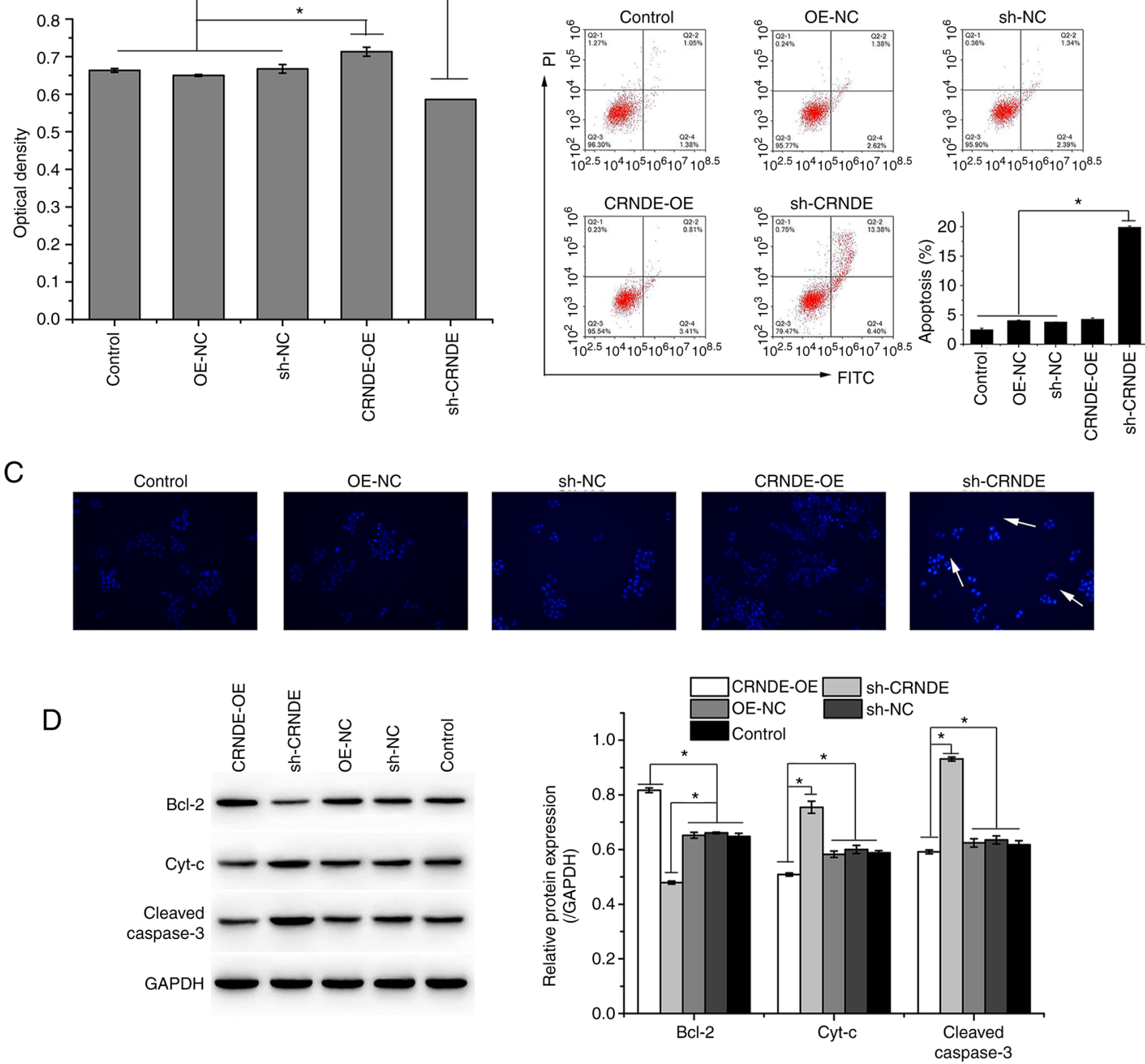

$\mathrm{E}$

$5 \mu \mathrm{M}$
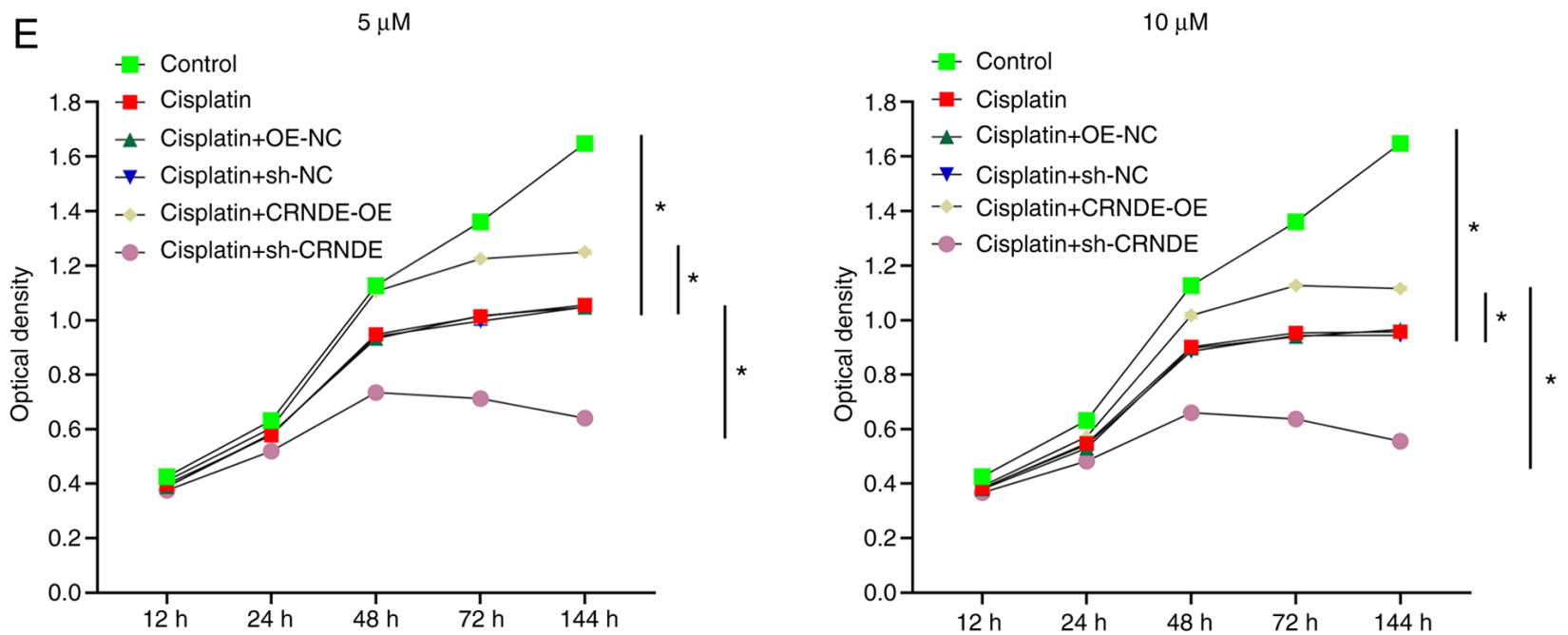

Figure 2. CRNDE silencing inhibits proliferation and promotes apoptosis and cisplatin sensitivity in HCT-116 cells. (A) The optical density of transfected HCT-116 cells was detected by a CCK-8 assay. Apoptosis in transfected HCT-116 cells was detected by (B) flow cytometry and (C) Hoechst-33258 staining (magnification: $\mathrm{x} 200$ ). (D) The expression of apoptosis-related proteins in transfected HCT-116 cells was detected by western blotting. (E) The optical density of transfected HCT-116 cells was detected after treatment with 5 or $10 \mu \mathrm{M}$ cisplatin. Data are presented as the mean $\pm \mathrm{SD}, \mathrm{n}=3,{ }^{*} \mathrm{P}<0.05$. 

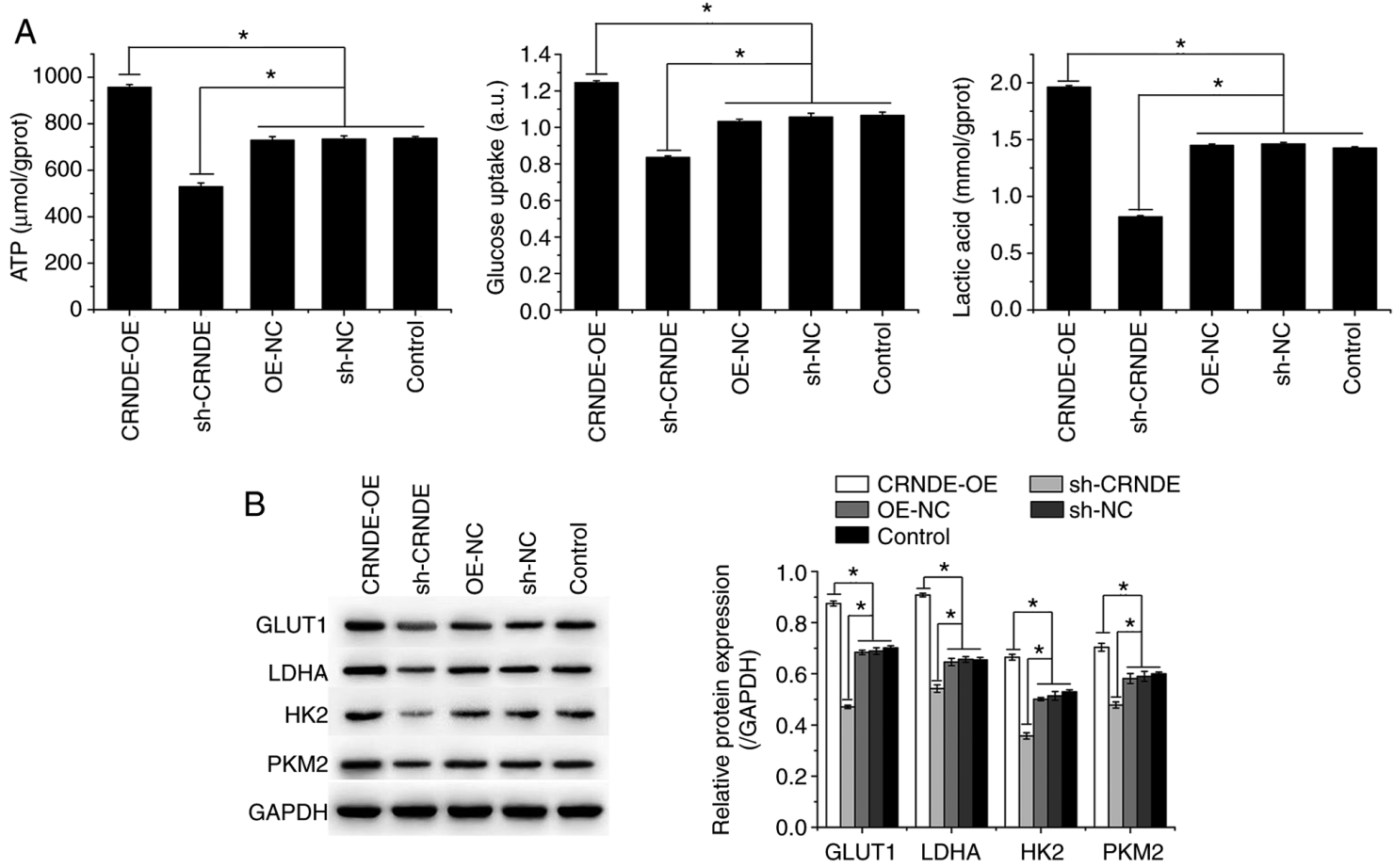

Figure 3. CRNDE silencing inhibits the Warburg effect in HCT-116 cells. (A) ATP production, lactic acid level, and glucose uptake in transfected HCT-116 cells were detected using biochemical assay. (B) The expression of GLUT1, LDHA, HK2, and PKM2 protein in transfected HCT-116 cells was detected by western blotting. Data are presented as the mean $\pm \mathrm{SD}, \mathrm{n}=3,{ }^{*} \mathrm{P}<0.05$.
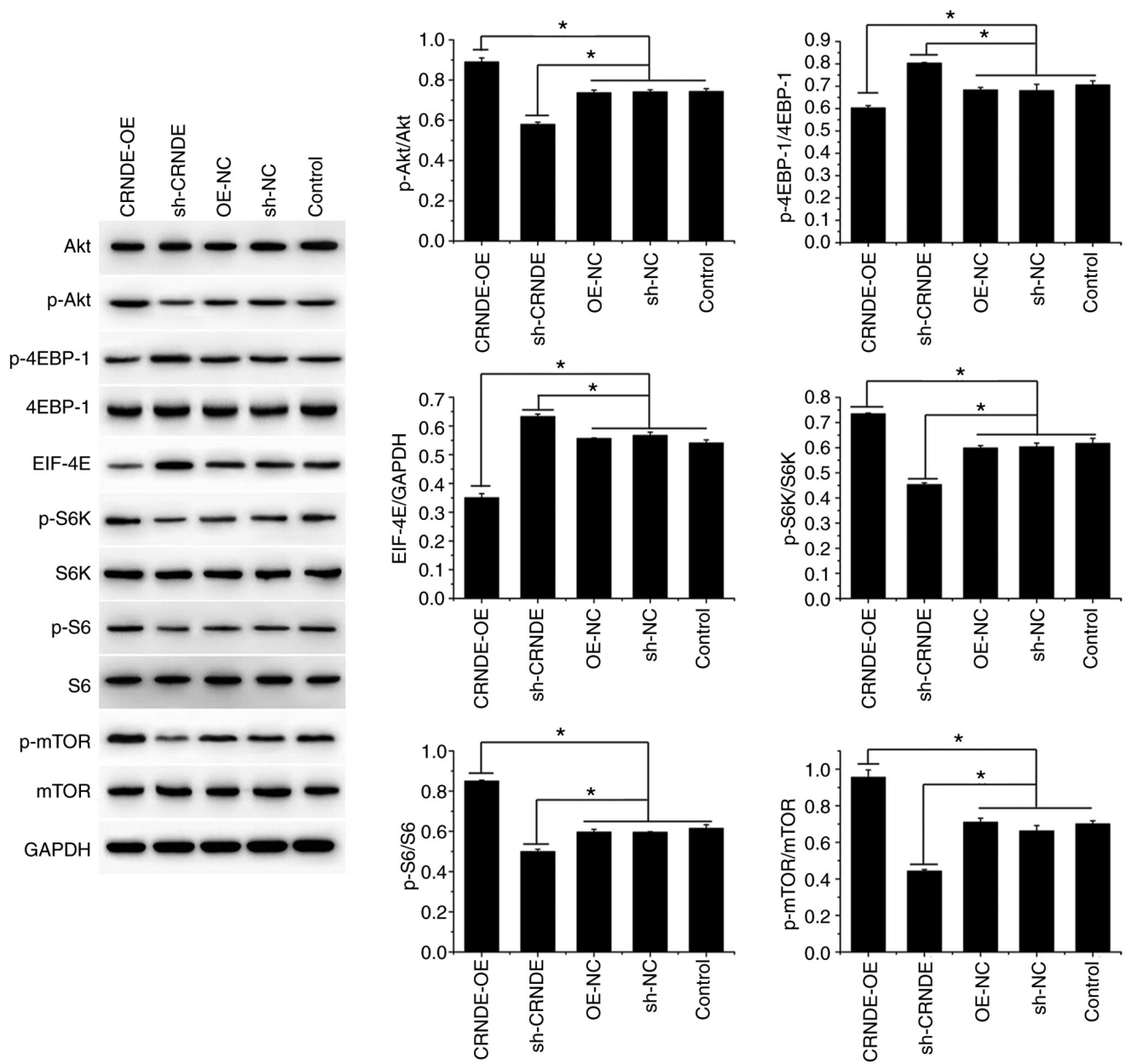

Figure 4. CRNDE silencing inhibits Akt/mTORC1 activation in HCT-116 cells. The protein expression of Akt, p-Akt, 4EBP-1, p-4EBP-1, EIF-4E, S6K, p-S6K, $\mathrm{S} 6, \mathrm{p}-\mathrm{S} 6, \mathrm{mTOR}$, and $\mathrm{p}-\mathrm{mTOR}$ in transfected HCT-116 cells was detected by western blotting. Data are presented as the mean $\pm \mathrm{SD}, \mathrm{n}=3$, $\mathrm{P}<0.05$. 


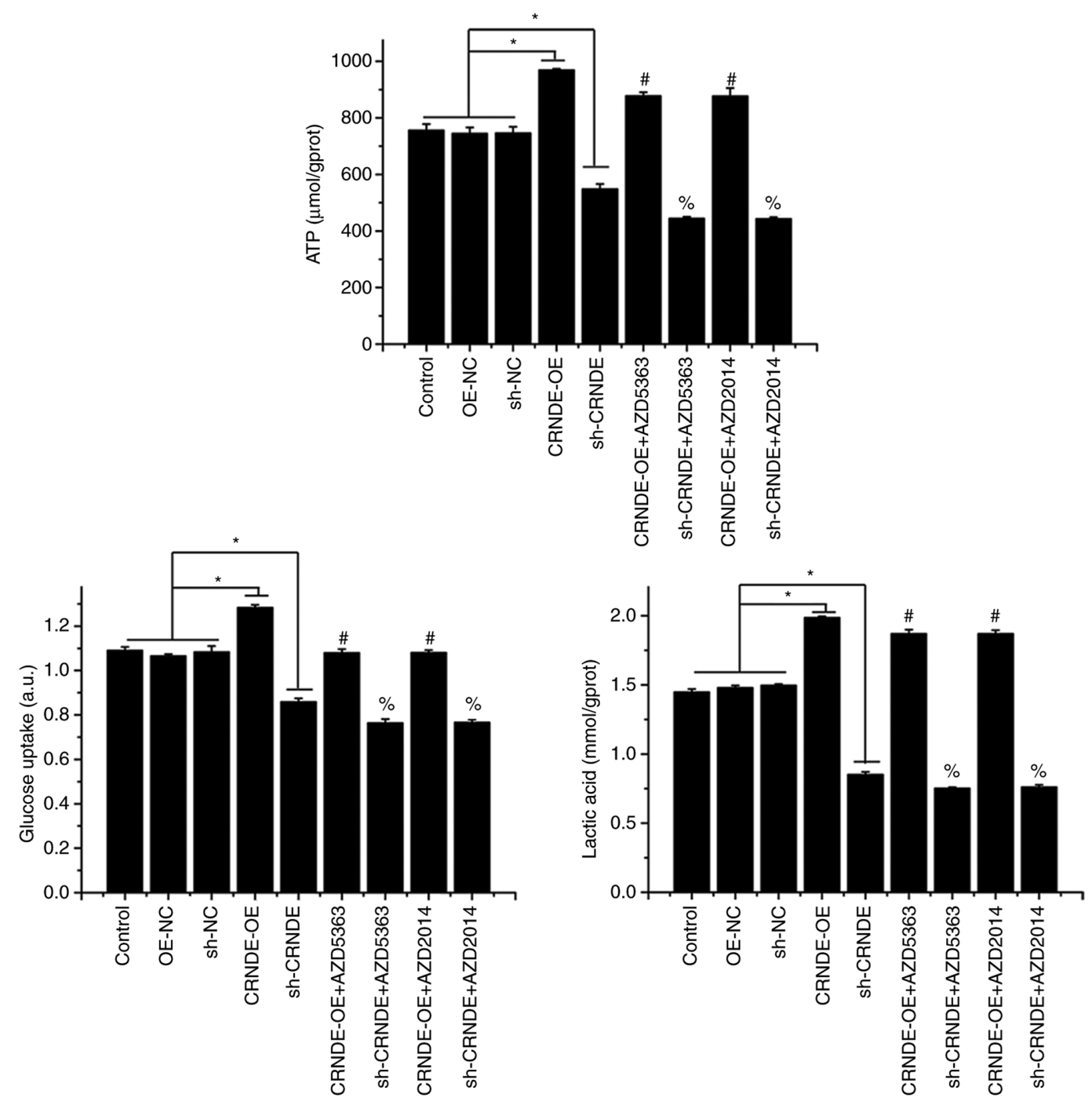

Figure 5. CRNDE silencing suppresses the Warburg effect in HCT-116 cells by inhibiting Akt/mTOR activation. ATP production, lactic acid levels, and glucose uptake in transfected HCT-116 cells following treatment with Akt and mTOR inhibitors were detected using corresponding biochemical assay kits. Data are presented as the mean $\pm \mathrm{SD}, \mathrm{n}=3$, AZD5363: Akt inhibitor, AZD2014: mTOR inhibitor, ${ }^{*} \mathrm{P}<0.05,{ }^{\#} \mathrm{P}<0.05$ vs. CRNDE, ${ }^{\text {\% }} \mathrm{P}<0.05$ vs. sh-CRNDE.

influence of the Akt/mTOR pathway on the regulatory effect of CRNDE on the Warburg effect in HCT-116 cells, Akt and mTOR were suppressed by corresponding inhibitors in transfected HCT-116 cells. As shown in Fig. 5, compared with the CRNDE-OE group, ATP production, lactic acid levels, and glucose uptake decreased in the CRNDE-OE+AZD5363 and CRNDE-OE+AZD2014 groups. In addition, the decrease in ATP production, lactic acid levels, and glucose uptake in the sh-CRNDE group were further decreased by Akt and mTOR inhibition. These results indicated that the effect of CRNDE on the Warburg effect in HCT-116 cells may be mediated by the Akt/mTOR pathway.

\section{Discussion}

In general, normal cells use mitochondria to oxidize glucose molecules as their main energy metabolism pathway. Glucose forms pyruvate through multi-step glycolysis. Pyruvic acid enters the mitochondria and is oxidized through the tricarboxylic acid cycle to produce ATP to meet the energy needs of cells (31). However, in tumor cells, pyruvic acid produced by glycolysis does not enter the mitochondria but is reduced to lactic acid by $\mathrm{LDH}$, even in the presence of sufficient oxygen (31). This phenomenon, known as the Warburg effect, is a form of metabolic reprogramming closely associated with cancer occurrence and development (32). Although less ATP is produced by glycolysis than by oxidative phosphorylation, glycolysis intermediates provide the carbon sources needed for rapid cell proliferation (33). In addition, lactic acid generated during glycolysis decreases the $\mathrm{pH}$ value of the extracellular matrix (34), whereas acidic microenvironments promote tumor invasion, metastasis, and radiotherapy resistance $(35,36)$. Glucose uptake is the first step of the Warburg effect, during which specific membrane transporters such as GLUTs are required (37). This glucose is then phosphorylated by HK to generate glucose-6-phosphate, which is subsequently converted into phosphoenol pyruvate (38), which is then converted into pyruvate by $\mathrm{PK}$, and the pyruvate is finally converted to lactic acid by LDH (39), which is the last step of glycolysis. Inhibiting the activity of GLUTs, HK, PK, and LDH has been shown to suppress the Warburg effect in cancer cells $(40,41)$, thereby modulating their apoptosis, proliferation, 
migration, invasion, and drug resistance (42-45). The current study indicated that CRNDE inhibition decreased the levels of ATP and lactic acid, glucose uptake, and the expression of GLUT1, LDHA, HK2, and PKM2 in HCT-116 cells. In addition, CRNDE inhibition suppressed the proliferation of HCT-116 cells while promoting their apoptosis and cisplatin sensitivity, which may be associated with its inhibitory action on the Warburg effect.

Additionally, the current study indicated that CRNDE inhibition decreases the activity of Akt/mTORC1, as demonstrated by the decrease in the protein expression of $\mathrm{p}-\mathrm{Akt}, \mathrm{p}-\mathrm{S} 6 \mathrm{~K}$, $\mathrm{p}-\mathrm{S} 6$, and $\mathrm{p}-\mathrm{mTOR}$ and the increase in the protein expression of p-4EBP-1 and EIF-4E. The activation of mTOR is associated with a series of proteins involved in two structurally and functionally distinct complexes, mTORC1 and mTORC2 (46). Of note, mTORC1 is regulated by Akt via phosphorylation at Ser 2448 (47). The Akt/mTORC1 pathway and its downstream factors are involved in the regulation of cellular metabolism. mTORC1, a central activator of the Warburg effect, has been shown to induce glycolytic enzymes in tumor cells (48). p70S6K is an effector of mTORC1 that promotes glucose metabolism from glycolysis to pentose phosphate, which in turn enhances cancer cell growth $(49,50)$. Another characterized downstream factor of mTORC1 is 4EBP1, which attenuates the initiation of protein translation by binding and inactivating EIF-4E. Previous findings have shown that 4EBP1 inhibition, regulated by mTOR activation, promotes glucose uptake and glycolysis (51). The suppression of Akt/mTORC1 activation downregulates the expression of GLUT1 and HK2 and inhibits glucose uptake, subsequently impairing glucose metabolism (52). In addition, the current study found that the increase in ATP and lactic acid levels and glucose uptake in HCT-116 cells induced by CRNDE overexpression was counteracted by Akt and mTOR inhibition, indicating that the effect of CRNDE on the Warburg effect in HCT-116 cells may be mediated by the Akt/mTORCl pathway.

In conclusion, the present study provides evidence demonstrating that CRNDE inhibition suppresses the Warburg effect in HCT-116 cells, in turn inhibiting their proliferation and promoting their apoptosis and cisplatin sensitivity. The mechanism underlying the action of CRNDE silencing may be the inactivation of $\mathrm{Akt} / \mathrm{mTORC} 1$ signaling. In vivo experiments are to be designed in follow-up studies to verify the findings of the current study.

\section{Acknowledgements}

Not applicable.

\section{Funding}

The present study was supported by the $\mathrm{PhD}$-initiated funding project of Hubei University of Chinese Medicine (grant no. 10110428).

\section{Availability of data and materials}

The datasets used and/or analyzed during the current study are available from the corresponding author on reasonable request.

\section{Authors' contributions}

WY and XY contributed to conception of the study. WY, YW and $X Y$ designed and performed the experiments, analyzed the data and drafted the manuscript. CT, YL and SC analyzed the data and provided technical support. All authors have read and approved the final manuscript. WY, YW, CT, YL, SC and $\mathrm{XY}$ confirm the authenticity of all the raw data.

\section{Ethics approval and consent to participate}

Not applicable.

\section{Patient consent for publication}

Not applicable.

\section{Competing interests}

The authors declare that they have no competing interests.

\section{References}

1. Chen W, Zheng R, Baade PD, Zhang S, Zeng H, Bray F, Jemal A, Yu XQ and He J: Cancer statistics in China, 2015. CA Cancer J Clin 66: 115-132, 2016.

2. Bray F, Ferlay J, Soerjomataram I, Siegel RL, Torre LA and Jemal A: Global cancer statistics 2018: GLOBOCAN estimates of incidence and mortality worldwide for 36 cancers in 185 countries. CA Cancer J Clin 68: 394-424, 2018.

3. Binefa G, Rodriguez-Moranta F, Teule A and Medina-Hayas M: Colorectal cancer: From prevention to personalized medicine. World J Gastroenterol 20: 6786-6808, 2014.

4. Brenner H, Kloor M and Pox CP: Colorectal cancer. Lancet 383: 1490-1502, 2014

5. Kim SE, Paik HY, Yoon H, Lee JE, Kim N and Sung MK: Sexand gender-specific disparities in colorectal cancer risk. World J Gastroenterol 21: 5167-5175, 2015.

6. Woolston A, Khan K, Spain G, Barber LJ, Griffiths B, Gonzalez-Exposito R, Hornsteiner L, Punta M, Patil Y, Newey A, et al: Genomic and transcriptomic determinants of therapy resistance and immune landscape evolution during Anti-EGFR treatment in colorectal cancer. Cancer Cell 36: 35-50 e9, 2019.

7. Marjaneh RM, Khazaei M, Ferns GA, Avan A and Aghaee-Bakhtiari SH: The role of microRNAs in 5-FU resistance of colorectal cancer: Possible mechanisms. J Cell Physiol 234: 2306-2316, 2019.

8. Wei L, Wang X, Lv L, Liu J, Xing H, Song Y, Xie M, Lei T, Zhang $\mathrm{N}$ and Yang $\mathrm{M}$ : The emerging role of microRNAs and long noncoding RNAs in drug resistance of hepatocellular carcinoma. Mol Cancer 18: 147, 2019.

9. Xiong G, Liu C, Yang G, Feng M, Xu J, Zhao F, You L, Zhou L, Zheng L, Hu Y, et al: Long noncoding RNA GSTM3TV2 upregulates LAT2 and OLR1 by competitively sponging let-7 to promote gemcitabine resistance in pancreatic cancer. J Hematol Oncol 12: 97, 2019.

10. Martens-Uzunova ES, Bottcher R, Croce CM, Jenster G, Visakorpi $\mathrm{T}$ and Calin GA: Long noncoding RNA in prostate, bladder, and kidney cancer. Eur Urol 65: 1140-1151, 2014.

11. Ni W, Yao S, Zhou Y, Liu Y, Huang P, Zhou A, Liu J, Che L and Li J: Long noncoding RNA GAS5 inhibits progression of colorectal cancer by interacting with and triggering YAP phosphorylation and degradation and is negatively regulated by the $\mathrm{m}^{6} \mathrm{~A}$ reader YTHDF3. Mol Cancer 18: 143, 2019.

12. Fu D, Lu C, Qu X, Li P, Chen K, Shan L and Zhu X: LncRNA TTN-AS1 regulates osteosarcoma cell apoptosis and drug resistance via the miR-134-5p/MBTD1 axis. Aging (Albany NY) 11: 8374-8385, 2019.

13. Fang Z, Zhao J, Xie W, Sun Q, Wang H and Qiao B: LncRNA UCA 1 promotes proliferation and cisplatin resistance of oral squamous cell carcinoma by sunppressing miR-184 expression. Cancer Med 6: 2897-2908, 2017. 
14. Wang $\mathrm{P}, \mathrm{Xu}$ J, Wang $\mathrm{Y}$ and Cao X: An interferon-independent lncRNA promotes viral replication by modulating cellular metabolism. Science 358: 1051-1055, 2017.

15. Zhang J, Yin M, Peng G and Zhao Y: CRNDE: An important oncogenic long non-coding RNA in human cancers. Cell Prolif 51: e12440, 2018.

16. Han P, Li JW, Zhang BM, Lv JC, Li YM, Gu XY, Yu ZW, Jia YH, Bai XF, Li L, et al: The lncRNA CRNDE promotes colorectal cancer cell proliferation and chemoresistance via miR-181a-5p-mediated regulation of $\mathrm{Wnt} / \beta$-catenin signaling. Mol Cancer 16: 9, 2017.

17. Ji D, Jiang C, Zhang L, Liang N, Jiang T, Yang B and Liang $\mathrm{H}$ : LncRNA CRNDE promotes hepatocellular carcinoma cell proliferation, invasion, and migration through regulating miR-203/BCAT1 axis. J Cell Physiol 234: 6548-6560, 2019.

18. Jing H, Xia H, Qian M and Lv X: Long noncoding RNA CRNDE promotes non-small cell lung cancer progression via sponging microRNA-338-3p. Biomed Pharmacother 110: $825-833,2019$

19. Liang $\mathrm{C}$, Zhang $\mathrm{B}, \mathrm{Ge} \mathrm{H}, \mathrm{Xu} \mathrm{Y}, \mathrm{Li} \mathrm{G}$ and $\mathrm{Wu}$ J: Long non-coding RNA CRNDE as a potential prognostic biomarker in solid tumors: A meta-analysis. Clin Chim Acta 481: 99-107, 2018.

20. Ding C, Han F, Xiang H, Xia X, Wang Y, Dou M, Zheng J, Li Y, Xue W, Ding X and Tian P: LncRNA CRNDE is a biomarker for clinical progression and poor prognosis in clear cell renal cell carcinoma. J Cell Biochem 119: 10406-10414, 2018.

21. Yang HY, Huang CP, Cao MM, Wang YF and Liu Y: Long non-coding RNA CRNDE may be associated with poor prognosis by promoting proliferation and inhibiting apoptosis of cervical cancer cells through targeting PI3K/AKT. Neoplasma 65 872-880, 2018

22. Sun XH, Fan WJ, An ZJ and Sun Y: Inhibition of long noncoding RNA CRNDE increases chemosensitivity of medulloblastoma cells by targeting miR-29c-3p. Oncol Res 28: 95-102, 2020.

23. Ding J, Li J, Wang H, Tian Y, Xie M, He X, Ji H, Ma Z, Hui B, Wang K and Ji G: Long noncoding RNA CRNDE promotes colorectal cancer cell proliferation via epigenetically silencing DUSP5/CDKN1A expression. Cell Death Dis 8 : e2997, 2017.

24. Sun F, Liang W and Qian J: The identification of CRNDE, H19, UCA1 and HOTAIR as the key lncRNAs involved in oxaliplatin or irinotecan resistance in the chemotherapy of colorectal cancer based on integrative bioinformatics analysis. Mol Med Rep 20: 3583-3596, 2019

25. Gao H, Song X, Kang T, Yan B, Feng L, Gao L, Ai L, Liu X, $\mathrm{Yu} \mathrm{J}$ and $\mathrm{Li} \mathrm{H}$ : Long noncoding RNA CRNDE functions as a competing endogenous RNA to promote metastasis and oxaliplatin resistance by sponging miR-136 in colorectal cancer. Onco Targets Ther 10: 205-216, 2017.

26. Wiese EK and Hitosugi T: Tyrosine Kinase signaling in cancer metabolism: PKM2 Paradox in the Warburg Effect. Front Cell Dev Biol 6: 79, 2018.

27. Li L, Liang Y, Kang L, Liu Y, Gao S, Chen S, Li Y, You W, Dong Q, Hong T, et al: Transcriptional regulation of the warburg effect in cancer by SIX1. Cancer Cell 33: 368-385.e7, 2018.

28. Li J, Cheng D, Zhu M, Yu H, Pan Z, Liu L, Geng Q, Pan H, Yan M and Yao M: OTUB2 stabilizes U2AF2 to promote the Warburg effect and tumorigenesis via the AKT/mTOR signaling pathway in non-small cell lung cancer. Theranostics 9: 179-195, 2019.

29. Yang $\mathrm{Z}$ and Chen $\mathrm{W}$ : Long non-coding RNA CRNDE promote the progression of tongue squamous cell carcinoma through regulating the PI3K/AKT/mTOR signaling pathway. RSC Adv 9: 21381-21390, 2019.

30. Livak KJ and Schmittgen TD: Analysis of relative gene expression data using real-time quantitative PCR and the 2(-Delta Delta C(T)) Method. Methods 25: 402-408, 2001

31. Lunt SY and Vander Heiden MG: Aerobic glycolysis: Meeting the metabolic requirements of cell proliferation. Annu Rev Cell Dev Biol 27: 441-464, 2011.

32. Vaupel P, Schmidberger H and Mayer A: The Warburg effect: Essential part of metabolic reprogramming and central contributor to cancer progression. Int J Radiat Biol 95: 912-919, 2019.

33. Brooks GA: Cell-cell and intracellular lactate shuttles. J Physiol 587(Pt 23): 5591-5600, 2009.
34. Held-Warmkessel J and Dell DD: Lactic acidosis in patients with cancer. Clin J Oncol Nurs 18: 592-594, 2014.

35. Peppicelli S, Bianchini F and Calorini L: Extracellular acidity, a 'reappreciated' trait of tumor environment driving malignancy: Perspectives in diagnosis and therapy. Cancer Metastasis Rev 33: 823-832, 2014

36. Shiraishi T, Verdone JE, Huang J, Kahlert UD, Hernandez JR, Torga G, Zarif JC, Epstein T, Gatenby R, McCartney A, et al: Glycolysis is the primary bioenergetic pathway for cell motility and cytoskeletal remodeling in human prostate and breast cancer cells. Oncotarget 6: 130-143, 2015.

37. Holman GD: Chemical biology probes of mammalian GLUT structure and function. Biochem J 475: 3511-3534, 2018.

38. Al-Azzam N: Sirtuin 6 and metabolic genes interplay in Warburg effect in cancers. J Clin Biochem Nutr 66: 169-175, 2020.

39. Vander Heiden MG, Cantley LC and Thompson CB: Understanding the Warburg effect: The metabolic requirements of cell proliferation. Science 324: 1029-1033, 2009.

40. Hong X, Zhong L, Xie Y, Zheng K, Pang J, Li Y, Yang Y, Xu X, Mi P, Cao H, et al: Matrine reverses the warburg effect and suppresses colon cancer cell growth via negatively regulating HIF-1 $\alpha$. Front Pharmacol 10: 1437, 2019.

41. Yu W, Yang Z, Huang R, Min Z and Ye M: SIRT6 promotes the Warburg effect of papillary thyroid cancer cell BCPAP through reactive oxygen species. Onco Targets Ther 12: 2861-2868, 2019.

42. Lu Z, Guo Y, Zhang X, Li J, Li L, Zhang S and Shan C: ORY-1001 Suppresses cell growth and induces apoptosis in lung cancer through triggering HK2 mediated warburg effect. Front Pharmacol 9: 1411, 2018.

43. Lin G, Wu Y, Cai F, Li Z, Su S, Wang J, Cao J and Ma L: Matrine promotes human myeloid leukemia cells apoptosis through warburg effect mediated by hexokinase 2 . Front Pharmacol 10: 1069, 2019.

44. Wang S, Zhang Y, Cai Q, Ma M, Jin LY, Weng M, Zhou D, Tang Z, Wang JD and Quan Z: Circular RNA FOXP1 promotes tumor progression and Warburg effect in gallbladder cancer by regulating PKLR expression. Mol Cancer 18: 145, 2019.

45. Icard P, Shulman S, Farhat D, Steyaert JM, Alifano M and Lincet H: How the Warburg effect supports aggressiveness and drug resistance of cancer cells? Drug Resist Updat 38: 1-11, 2018.

46. Lu Z, Shi X, Gong F, Li S, Wang Y, Ren Y, Zhang M, Yu B, Li Y, Zhao W, et al: RICTOR/mTORC2 affects tumorigenesis and therapeutic efficacy of mTOR inhibitors in esophageal squamous cell carcinoma. Acta Pharm Sin B 10: 1004-1019, 2020.

47. Chen GQ, Tang CF, Shi XK, Lin CY, Fatima S, Pan XH, Yang DJ, Zhang G, Lu AP, Lin SH and Bian ZX: Halofuginone inhibits colorectal cancer growth through suppression of Akt/mTORC1 signaling and glucose metabolism. Oncotarget 6: 24148-24162, 2015.

48. Sun Q, Chen X, Ma J, Peng H, Wang F, Zha X, Wang Y, Jing Y, Yang H, Chen R, et al: Mammalian target of rapamycin up-regulation of pyruvate kinase isoenzyme type M2 is critical for aerobic glycolysis and tumor growth. Proc Natl Acad Sci USA 108: 4129-4134, 2011.

49. Duvel K, Yecies JL, Menon S, Raman P, Lipovsky AI, Souza AL, Triantafellow E, Ma Q, Gorski R, Cleaver S, et al: Activation of a metabolic gene regulatory network downstream of mTOR complex 1. Mol Cell 39: 171-183, 2010.

50. Yalcin S, Marinkovic D, Mungamuri SK, Zhang X, Tong W, Sellers R and Ghaffari S: ROS-mediated amplification of AKT/mTOR signalling pathway leads to myeloproliferative syndrome in Foxo3(-/-) mice. EMBO J 29: 4118-4131, 2010.

51. Maiese K, Chong ZZ, Shang YC and Wang S: mTOR: On target for novel therapeutic strategies in the nervous system. Trends Mol Med 19: 51-60, 2013.

52. Siska PJ, van der Windt GJ, Kishton RJ, Cohen S, Eisner W, MacIver NJ, Kater AP, Weinberg JB and Rathmell JC: Suppression of glut 1 and glucose metabolism by decreased $\mathrm{Akt} / \mathrm{mTORC1}$ signaling drives $\mathrm{T}$ cell impairment in $\mathrm{B}$ cell leukemia. J Immunol 197: 2532-2540, 2016.

This work is licensed under a Creative Commons Attribution-NonCommercial-NoDerivatives 4.0 International (CC BY-NC-ND 4.0) License. 